\title{
Preferential selection of variants in ferromagnetic Fe-Pd alloys under magnetic field
}

\author{
S. Farjami ${ }^{\mathrm{a}}$, T. Fukuda and T. Kakeshita \\ Department of Materials Science and Engineering, Graduate School of Engineering, \\ Osaka University, 2-1, Yamada-oka, Suita, Osaka 565-0871, Japan
}

\begin{abstract}
Effect of magnetic field on microstructure formation through a disorder-order transformation has been studied in $\mathrm{Fe}-$ Pd alloy. Single crystal of the disordered Fe-55at\%Pd is subjected to an ordering heat-treatment under a magnetic field. When the ordering heat-treatment is performed without applying a magnetic field, three lattice corresponding variants are formed equivalently. On the other hand, when the ordering heat-treatment is made under a magnetic field of $4 \mathrm{~T}$ and higher applied along the [001] direction of the disordered phase, a single variant with an easy axis along the field direction is obtained. A tweed microstructure has been observed at early stage of ordering with streaks in the $<011>$ directions. High density of antiphase boundary after formation of a single variant indicates the formation of homogeneous nucleation of the ordered $\mathrm{L}_{0}$ phase in a disordered fcc matrix at the early stage of ordering.
\end{abstract}

\section{Introduction}

Magnetic field-induced strain due to the rearrangement of martensite variants in some ferromagnetic shape memory alloys has attracted much attention since it was first found in $\mathrm{Ni}_{2} \mathrm{MnGa}$ in 1996 and the condition for the occurrence of the rearrangement is now well understood quantitatively [1-4]. According to the studies, the rearrangement of martensite variants by magnetic field is essentially related to its large magnetocrystalline anisotropy. Considering the effectiveness of magnetocrystalline anisotropy on evolution of morphology, we can expect selected formation of a variant even in a diffusive process under magnetic field when the product phase is ferromagnetic and has a large magnetocrystalline anisotropy. As for such transformations, a disorder-order transformation in an Fe-Pd can be considered. The reason is as follows.

Near-equiatomic Fe-Pd alloy transforms from a disordered A1-type (cubic) structure to an ordered $\mathrm{L}_{0^{-}}$ type (tetragonal) structure and the tetragonal phase has three variants. The ordered phase has high Curie temperature, and has a large magnetocrystalline anisotropy, where the easy axis is the $c$-axis and the hard axis is the $a$-axis [5-8]. Therefore, there arises a significant energy difference among the variants under a magnetic field, and the variant with the lowest magnetic energy is expected to form selectively during the disorder-order transformation.

In this study we will focus on the effect of magnetic field on microstructure formation during a disorder-order transformation in Fe-55at\%Pd single crystal. To determine the influence of a magnetic field on microstructure formation we have carried out a two-step ordering heat-treatment. The first step of ordering is made under a magnetic field of up to $10 \mathrm{~T}$ applied along the $[001]_{\mathrm{A} 1}$ direction to initiate the ordering under the magnetic field. This is followed by a second step of ordering without using a magnetic field that would result in a disorder-order transformation. We have also studied the microstructure formed through the disorder-order transformation under a magnetic field by transmission electron microscopy (TEM).

\section{Experimental procedure}

An ingot of $\mathrm{Fe}-55 \mathrm{at} \% \mathrm{Pd}$ was prepared by arc melting. A single crystalline rod was grown by a floating zone method and then homogenized at $1273 \mathrm{~K}$ for $168 \mathrm{~h}$ followed by iced-water quenching. Crystallographic orientation of the single crystal was determined by using a back reflection Laue method. Cubic specimens with each edge parallel to the $\langle 001\rangle_{\mathrm{A} 1}$ direction were cut out. After cutting, the specimens were heat-treated at

\footnotetext{
a e-mail: farjami@mat.eng.osaka-u.ac.jp
} 
$1173 \mathrm{~K}$ for $1 \mathrm{~h}$ followed by iced-water quenching to obtain the disordered phase. Ordering heat-treatment under a magnetic field was made by using a furnace inserted into a superconducting magnet. The specimen is located at the center of the hot zone which is also the center of the magnetic field.

\section{Results and discussion}

Figure 1 shows an electron diffraction pattern of as-quenched (from $1173 \mathrm{~K}$ ) specimen taken at room temperature using [100] beam direction. In addition to fundamental reflection of the fcc structure, we can observe very weak (001) superlattice reflections (SLRs) in the diffraction pattern probably due to the short range ordering. No specific microstructure can be observed at this stage. From these characteristics, it is most likely that almost disordered state is obtained in the present specimen by quenching from $1173 \mathrm{~K}$.

The characteristic temperatures such as the Curie temperature and the disorder-order transformation temperature have been determined by resistivity measurement. Temperature dependence of resistivity during heating and cooling processes is shown in Fig. 2. In order to evaluate the Curie temperature of the ordered phase, the disordered specimen was heat-treated at $773 \mathrm{~K}$ for $24 \mathrm{~h}$ (which is described later) to obtain a highly ordered state. Then, resistivity has been measured in the heating process from room temperature up to $1200 \mathrm{~K}$ with a heating rate of $1 \mathrm{~K} / \mathrm{min}$ shown by solid circles in Fig. 2. The resistivity heating curve exhibits a bending at $670 \mathrm{~K}$ which corresponds to the Curie temperature of the ordered phase, $T_{\mathrm{c}}^{(\mathrm{o})}$. On further heating, the resistivity increases gradually and then decreases sharply due to the transformation from the ordered phase to the disordered phase. The order-disorder transformation temperature, $T^{(\mathrm{o}-\mathrm{d})}$ is about $1025 \mathrm{~K}$. Then, the specimen is cooled down from $1200 \mathrm{~K}$ to room temperature with a cooling rate of $1 \mathrm{~K} / \mathrm{min}$. The corresponding cooling curve is shown by open circles. The disorder-order transformation temperature, $T^{(\mathrm{d}-\mathrm{o})}$, is shown as a sharp increase in resistivity cooling curve at $955 \mathrm{~K}$. Considering the characteristic temperatures, we have determined the ordering temperature of the two-step ordering heat-treatment as a trial: the first step of ordering at $673 \mathrm{~K}$ (close to the Curie temperature) and the second step of ordering at $773 \mathrm{~K}$ (below the disorder-order transformation temperature).

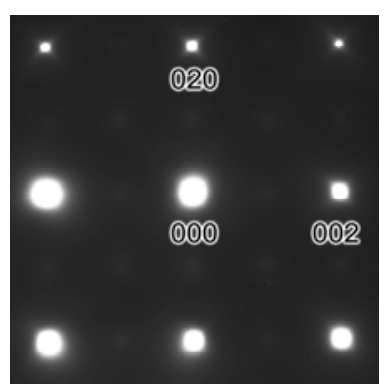

Fig. 1. Electron diffraction pattern of as-quenched specimen from $1173 \mathrm{~K}$.

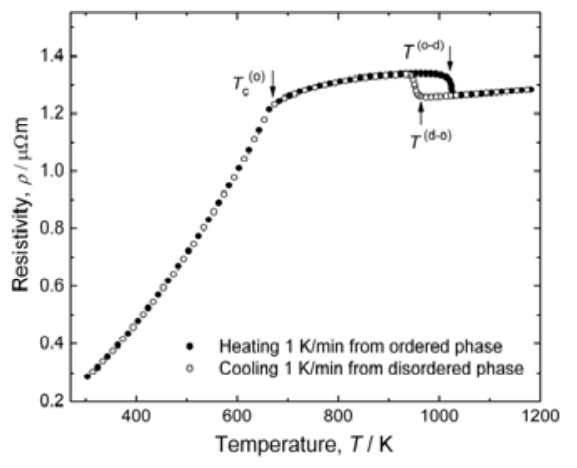

Fig. 2. Temperature dependence of the electrical resistivity.

To know the progress of ordering at the selected ordering temperature of the first step $(673 \mathrm{~K})$ and second step $(773 \mathrm{~K})$, time evolution of the disorder-order transformation has been examined by a conventional method. That is, we measure the electrical resistivity at $273 \mathrm{~K}$ after performing an isothermal heat-treatment at the set temperature followed by quenching into iced-water. The result is shown in Fig. 3, where the resistivity is normalized by the value of the as-quenched specimen, $\rho_{\text {A.Q. }}$ at $273 \mathrm{~K}$. The figure indicates that the resistivity saturates within $24 \mathrm{~h}$ at $773 \mathrm{~K}$, while not within $160 \mathrm{~h}$ at $673 \mathrm{~K}$. We have decided the ordering period of the first step to be $1 \mathrm{~h}$ to realize the early stage of ordering. This ordering period of $1 \mathrm{~h}$ is indicated by an arrow in the figure, where there is a small change in the resistivity due to the progress of ordering. The ordering period of the second step was selected to be $24 \mathrm{~h}$, where there is no significant change in the resistivity value, meaning that the disorder-order transformation is almost completed. After the second step of ordering for $24 \mathrm{~h}$, the degree of order is obtained to be 0.94 by XRD measurement.

Figure 4(a) shows electron diffraction pattern after the first step of ordering at $673 \mathrm{~K}$ for $1 \mathrm{~h}$ without magnetic field. In the diffraction pattern, we can observe the SLRs of the three variants as indicated by arrows. This means that the three variants of the ordered phase are formed in the disordered fcc matrix after the first step of ordering. The dark field image in Fig. 4(b), taken using the reflection labeled 1 in Fig. 4(a), shows a fine dispersion of small ordered particles of about $1 \mathrm{~nm}$ aligned in the $<011>$ directions. This result clearly indicates that the ordering proceeds by the heat-treatment at $673 \mathrm{~K}$ for $1 \mathrm{~h}$. 


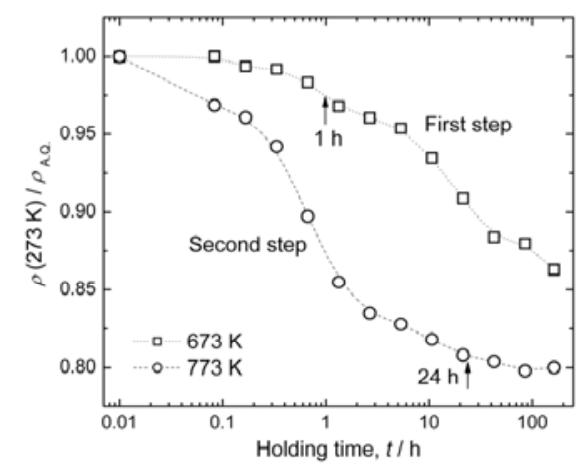

Fig. 3. Ordering time dependence of the electrical resistivity through isothermal annealing. The value of resistivity is normalized by resistivity of the asquenched specimen $\left(\rho_{\text {A. Q. }}\right)$ at $273 \mathrm{~K}$.

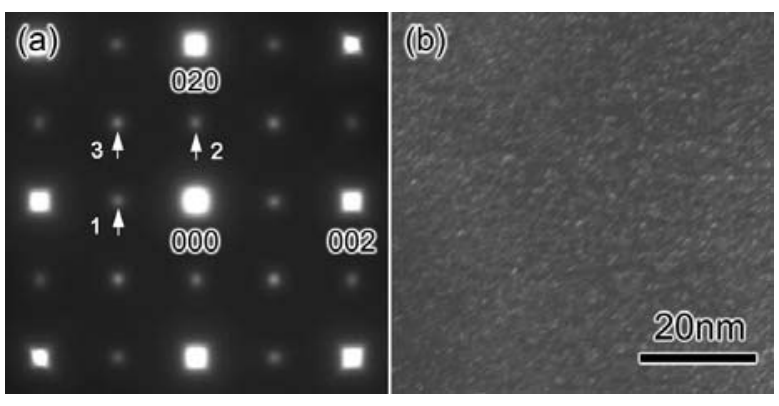

Fig. 4. Electron diffraction pattern (a) after the first step of ordering at $673 \mathrm{~K}$ for $1 \mathrm{~h}$ without magnetic field, (b) corresponding dark field image for the reflection labeled 1 in (a).

Magnetization curves measured at $300 \mathrm{~K}$ along the $\mathrm{X}-$, Y-, and Z-directions after the first step of ordering at $673 \mathrm{~K}$ for $1 \mathrm{~h}$ under magnetic fields of $10 \mathrm{~T}$ and $0 \mathrm{~T}$ are shown in Fig. 5(a) and (b), respectively and those after the second step of ordering at $773 \mathrm{~K}$ for $24 \mathrm{~h}$ are shown in Fig. 5(a') and ( $\left.\mathrm{b}^{\prime}\right)$, respectively. It is noted in Fig. 5(a) that after the first step of ordering, the Z-direction is clearly easy to magnetize compared to the Xand Y-directions when the applied magnetic field is $10 \mathrm{~T}$. This means that the fraction of the Z-variant, whose easy axis lies in the applied field direction, is larger than that of the X- and Y-variants. As known from Fig. 5(a'), the magnetization curve measured in the Z-direction saturates in a low field, but that in the X-and Y-directions increases linearly and saturate in the field range of $4 \mathrm{~T}$. This means that a single variant whose easy axis being parallel to the field direction is obtained. On the other hand, when the magnetic field is $0 \mathrm{~T}$, the difference in magnetization curves in the Z-, X-, and Y-directions becomes less significant after the first step of ordering, as seen in Fig. 5(b) and a single variant is not obtained after the second step of ordering, as seen in Fig. 5(b').

Electron diffraction pattern of the single variant described above is shown in Fig. 6(a). The direction of the applied magnetic field is shown by a horizontal arrow in Fig. 6. In the diffraction pattern, we can detect only the SLRs of the variant whose easy axis lies in the field direction (Z-variant). A bright field image in Fig. 6(b) shows a high density of antiphase boundary, due to the homogeneous nucleation of ordered $\mathrm{L}_{0}$ phase in the disordered fcc matrix at the early stage of ordering. Figure 6(c) is also a bright field image showing some thin plates along the $<011>$ directions. These thin plates are probably due to existence of very small fraction of the other two variants (X- and $\mathrm{Y}$-variants), although we can not detect their fractions by magnetization measurement (Fig. 5(a')).

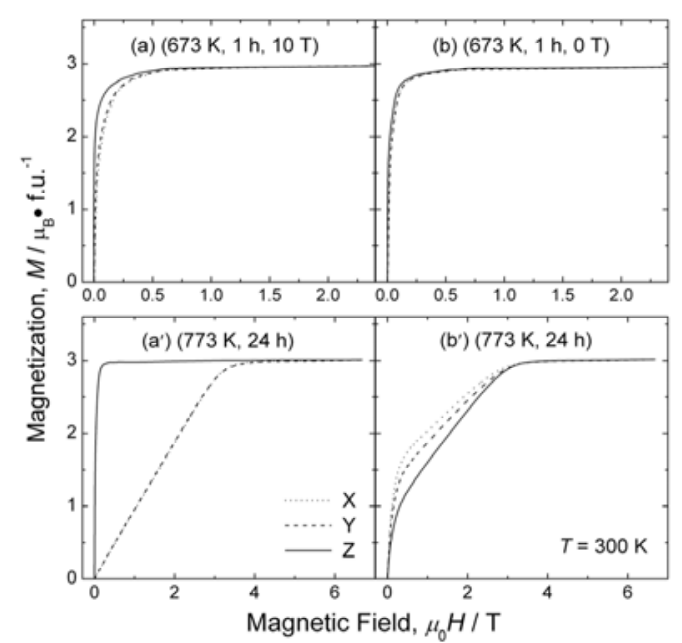

Fig. 5. Magnetization curves measured at $300 \mathrm{~K}$ along the X-, Y-, Z-directions after the first step of ordering at $673 \mathrm{~K}$ for $1 \mathrm{~h}$ under magnetic fields of $10 \mathrm{~T}$ (a) and $0 \mathrm{~T}$ (b); the second step of ordering at $773 \mathrm{~K}$ for $24 \mathrm{~h}$ without magnetic field (a'), (b').
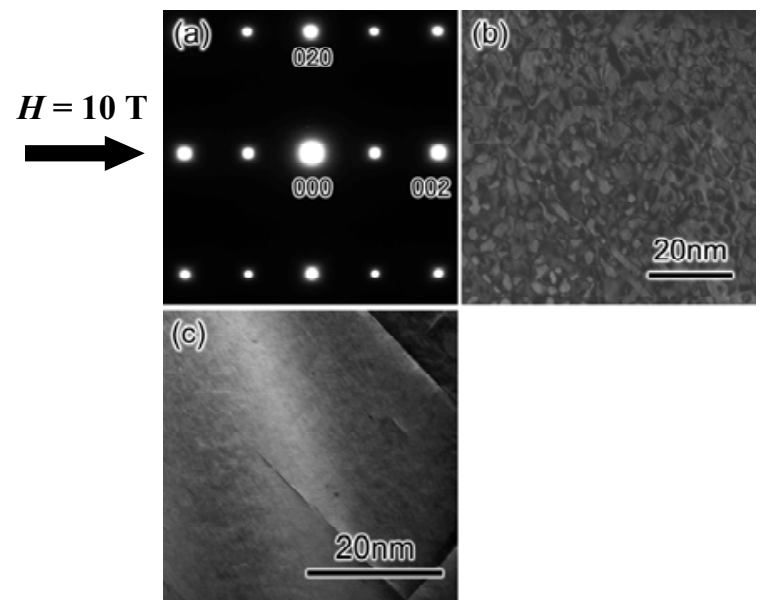

Fig. 6. Electron diffraction pattern of single variant (a) (to obtain the single variant, the first step is made at $673 \mathrm{~K}$ for $1 \mathrm{~h}$ under $10 \mathrm{~T}$ field and the second step at $773 \mathrm{~K}$ for $24 \mathrm{~h}$ without magnetic field). Bright field images obtained by exiting systematic 001 reflection (b) and 020 reflection (c). 


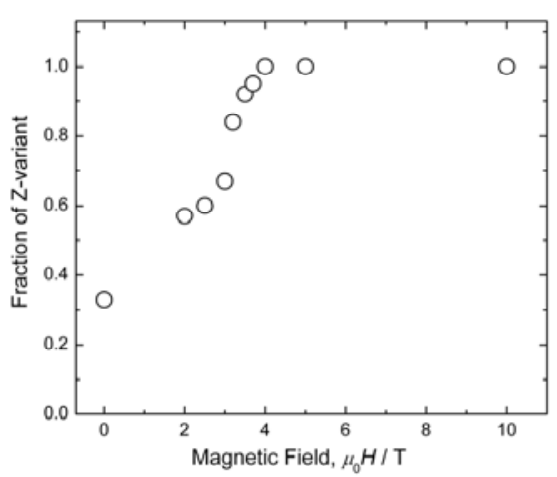

Fig. 7. Fraction of the Z-variant after the second step of ordering plotted as a function of magnetic field applied in the first step.

We have also investigated the effect of magnetic field-strength on the preferential selection of an ordered variant. That is, the first step of ordering has been carried out under different magnetic field-strengths of up to $10 \mathrm{~T}$. Figure 7 shows the fraction of the Z-variant, $f_{\mathrm{z}}$, after the second step of ordering as a function of magnetic field applied in the first step. The figure clearly shows that $f_{\mathrm{z}}$ increases with increasing magnetic field and reaches $100 \%$ at about $4 \mathrm{~T}$. We notice from the figure that there is a discontinuous increase in the $f_{\mathrm{z}}$ at about $3 \mathrm{~T}$, which indicates the existence of a critical magnetic field to obtain a single variant.

\section{Conclusions}

We have found the formation of a single variant of the ordered phase in Fe-55at\%Pd alloy by a two-step ordering heat-treatment; a magnetic field is applied in the first step of ordering while the second step is made without a magnetic field. The results suggest that the magnetic field is especially effective during the early stage of ordering for the selected formation of a preferred variant. The magnetic field-strength dependence of single variant formation indicates that there seems to be a critical magnetic field value required to obtain a single variant. A dark field image taken after the first step of ordering shows ordered particles of about $1 \mathrm{~nm}$ aligned in the $<011>$ directions. High density of antiphase boundary has been observed in a bright field image taken after formation of single variant.

\section{Acknowledgement}

This study was supported by Priority Assistance for the Formation of Worldwide Renowned Centers of Research - The Global COE Program (Project: Center of Excellence for Advanced Structural and Functional Materials Design) from the Ministry of Education, Culture, Sports, Science and Technology (MEXT), Japan. The authors would like to thank Prof. S. Nishijima of Osaka University for providing the superconducting magnet throughout the course of this work.

\section{References}

[1] K. Ullakko, J. K. Huang, C. Kantner, R. C. O'Handley and V. V. Kokorin, Appl. Phys. Lett. 69 (1996) 1966.

[2] R. D. James and M. Wuttig, Phil. Mag. A 77 (1998) 1273.

[3] K. Ullakko, J. K. Huang, V. V. Kokorin and R. C. O’Handley, Scr. Mater. 36 (1997) 1133.

[4] T. Sakamoto, T. Fukuda, T. Kakeshita, T. Takeuchi and K. Kishio, Mater. Trans. 44 (2003) 2495.

[5] G. M. Gushchin and F. N. Berseneva, Fiz. Met. Metalloved. 63 (1987) 926.

[6] B. Zhang, M. Lelovic and W. A. Soffa, Scr. Metall. Mater. 25 (1991) 1577.

[7] R. Oshima and H. Tokoro, J. Japan Inst. Metals 62 (1998) 317.

[8] L. Wang, D. E. Laughlin, Y. Wang and A. G. Khachaturyan, J. Appl. Phys. 93 (2003) 7984. 